PROCEEDINGS OF THE

AMERICAN MATHEMATICAL SOCIETY

Volume 128, Number 11, Pages 3311-3316

S 0002-9939(00)05710-5

Article electronically published on April 28, 2000

\title{
FINSLER METRICS AND ACTION POTENTIALS
}

\author{
RENATO ITURRIAGA AND HÉCTOR SÁNCHEZ-MORGADO
}

(Communicated by Michael Handel)

\begin{abstract}
We study the behavior of Mañé's action potential $\Phi_{k}$ associated to a convex superlinear Lagrangian, for $k$ bigger than the critical value $c(L)$. We obtain growth estimates for the action potential as a function of $k$. We also prove that the action potential can be written as $\Phi_{k}(x, y)=D_{F}(x, y)+$ $f(y)-f(x)$ where $f$ is a smooth function and $D_{F}$ is the distance function associated to a Finsler metric.
\end{abstract}

\section{INTRODUCTION}

Let $M$ be a closed riemannian manifold with riemannian metric $\langle v, v\rangle$. Consider the mechanical Lagrangian

$$
\begin{gathered}
L: T M \rightarrow \mathbb{R}, \\
(x, v) \mapsto \frac{1}{2}\langle v, v\rangle_{x}-U(x)
\end{gathered}
$$

where $U(x)$ is a differentiable function on $M$ called the potential.

It is well known that, on a fixed level of energy $e$, bigger than the maximum of $U$ the lagrangian flow is conjugate to the geodesic flow with metric $2(e-U(x))\langle v, v\rangle$. Moreover the reduced action of the Lagrangian is the distance for this metric. Either or both of these statements are known as the Maupertuis principle. See the books [1], 2] or [5].

Consider now a general convex superlinear Lagrangian $L: T M \rightarrow \mathbb{R}$. This means that $L$ restricted to each $T_{x} M$ has positive definite Hessian and

$$
\lim _{|v| \rightarrow \infty} \frac{L(x, v)}{|v|}=\infty
$$

uniformly on $x \in M$.

It was proven in 4 that for large energy values the lagrangian flow is conjugate to the flow of a Finsler metric. See below for the precise statement. In Theorem 1 we prove a generalization of the other statement of the Maupertuis principle. This was motivated by discussions with R. Montgomery about the presentation in [5], which also motivated Theorem 2 ,

Let $H: T^{*} M \rightarrow \mathbb{R}$ be the Hamiltonian associated to $L$ and let $\mathcal{L}: T M \rightarrow T^{*} M$ be the Legendre transform $(x, v) \mapsto \partial L / \partial v(x, v)$. Since $M$ is compact, the extremals of $L$ give rise to a complete flow $\varphi_{t}: T M \rightarrow T M$ called the Euler-Lagrange flow of

Received by the editors December 28, 1998.

2000 Mathematics Subject Classification. Primary 37J50, $70 \mathrm{H} 30$.

Both authors were partially supported by CONACYT-México grant \# 28489-E.

(C)2000 American Mathematical Society 
the Lagrangian. Using the Legendre transform we can push forward $\varphi_{t}$ to obtain another flow $\varphi_{t}^{*}$ which is the Hamiltonian flow of $H$ with respect to the canonical symplectic structure of $T^{*} M$. Recall that the energy $E: T M \rightarrow \mathbb{R}$ is defined by

$$
E(x, v)=\frac{\partial L}{\partial v}(x, v) \cdot v-L(x, v) .
$$

Since $L$ is autonomous, $E$ is a first integral of the flow $\varphi_{t}$.

Recall that the action of the Lagrangian $L$ on an absolutely continuous curve $\gamma:[a, b] \rightarrow M$ is defined by

$$
A_{L}(\gamma)=\int_{a}^{b} L(\gamma(t), \dot{\gamma}(t)) d t
$$

Given two points $x$ and $y$ in $M$ and $T>0$ denote by $\mathcal{C}_{T}(x, y)$ the set of absolutely continuous curves $\gamma:[0, T] \rightarrow M$, with $\gamma(0)=x$ and $\gamma(T)=y$. For each $k \in \mathbb{R}$ we define the action potential $\Phi_{k}: M \times M \rightarrow \mathbb{R}$ by

$$
\Phi_{k}(x, y)=\inf \left\{A_{L+k}(\gamma): \gamma \in \bigcup_{T>0} \mathcal{C}_{T}(x, y)\right\}
$$

The critical value of $L$, which was introduced by Mañé in [6], is the real number $c(L)$ defined as the infimum of $k \in \mathbb{R}$ such that for some $x \in M, \Phi_{k}(x, x)>-\infty$. For $k \geq c(L)$, we have that $\Phi_{k}(x, y)>-\infty$ for every $x, y$ and it is a Lipschitz function that satisfies the triangle inequality.

For any $k>c(L)$ the flow on the energy level $k$ is conjugate to the geodesic flow of an appropriately chosen Finsler metric on $M$ (see [4]).

Given a Finsler metric $\sqrt{F}$ and an absolutely continuous curve $\gamma$ we can define its Finsler length as

$$
l_{F}(\gamma)=\int \sqrt{F(\dot{\gamma})}
$$

Observe that since the Finsler metric is homogeneous of degree one, the definition does not depend on the parameterization of the curve. Finally we define the Finsler distance as

$$
D_{F}(x, y)=\inf \left\{l_{F}(\gamma)\right\}
$$

where the infimum is taken over all absolutely continuous curves joining $x$ and $y$.

Theorem 1. If $k$ is bigger than the critical value, then there exist a Finsler metric $\sqrt{F}$ and $a C^{\infty}$ real valued function $f$ on $M$ such that $\Phi_{k}(x, y)=D_{F}(x, y)+f(y)-$ $f(x)$. Moreover if $k$ is bigger than $-\inf L$, then we can choose $f=0$.

As a consequence of Theorem 1 we have that there is a neighborhood $V$ of the diagonal $\Delta$ in $M \times M$, such that $\Phi_{k}$ is differentiable in $V \backslash \Delta$.

For $x, y$ fixed and $T>0$ define

$$
S(T)=\inf \left\{A_{L}(\gamma): \gamma \in \mathcal{C}_{T}(x, y)\right\} .
$$

It is easily shown that $S(T)$ is continuous. Although $S(T)$ is not necessarily convex, its Legendre transform:

$$
S^{*}(e)=\max _{T>0}(e T-S(T))
$$


is a well defined convex function and coincides with the Legendre transform of the convex hull $\bar{S}$ of $S$. Notice that

$$
\Phi_{k}(x, y)=-S^{*}(-k)
$$

and so the domain of $S^{*}$ is $\operatorname{dom} S^{*}=(-\infty,-c(L)]$. It follows from the definition of the action potential that $g(k)=\Phi_{k}(x, y)$ is nondecreasing and so is $S^{*}$.

Theorem 2. For all $x, y$ in $M$ we have that:

(a) g grows slower than any linear function; that is,

$$
\lim _{k \rightarrow \infty} \frac{g(k)}{k}=0 .
$$

(b) The right derivative of $g$ at $c(L)$ is infinite.

(c) $\lim _{T \rightarrow \infty} S(T) / T=-c(L)$.

\section{Proofs}

Proof of Theorem [ It is well known that if $f$ is a convex function of a real variable, then

(1) If $x \in \operatorname{int}(\operatorname{dom} f)$, then both one side derivatives exist and $f_{-}^{\prime}(x) \leq f_{+}^{\prime}(x)$.

(2) If $x \in \operatorname{dom} f$ is a boundary point, then the corresponding one side derivative exists.

(3) If $x<y, f_{+}^{\prime}(x) \leq f_{-}^{\prime}(y)$.

Define

$$
\operatorname{rang} \partial f=\bigcup_{x \in \operatorname{dom} f}\left[f_{-}^{\prime}(x), f_{+}^{\prime}(x)\right]
$$

It is proved in [8], Section 24, that

$$
\operatorname{int}\left(\operatorname{dom} f^{*}\right) \subset \operatorname{rang} \partial f \subset \operatorname{dom} f^{*} .
$$

Therefore

$$
\operatorname{rang} \partial S^{*}=\operatorname{dom} S^{* *}=\operatorname{dom} \bar{S}=(0, \infty)
$$

Thus

$$
\lim _{e \rightarrow-\infty} \frac{S^{*}(e)}{e}=0
$$

and

$$
S_{-}^{*^{\prime}}(-c(L))=\lim _{e \rightarrow-c(L)} S_{-}^{*^{\prime}}(e)=\infty .
$$

From equation (1), items (a) and (b) follow.

By the same kind of arguments $\lim _{T \rightarrow \infty} \bar{S}(T) / T=-c(L)$, and then

$$
-c(L) \leq \liminf _{T \rightarrow \infty} \frac{S(T)}{T} .
$$

To prove the other inequality, let $\mu$ be an ergodic minimizing probability, that is, an invariant ergodic probability for the lagrangian flow $\varphi_{t}$ such that

$$
m:=\int L d \mu \leq \int L d \nu
$$


for any invariant probability $\nu$. Mather proved that such measures exist (see [7]). Let $\theta \in T M$ be a regular point for $\mu$, such that

$$
\lim _{T \rightarrow \infty} \frac{1}{T} \int_{0}^{T} L\left(\varphi_{t}(\theta)\right) d t=m
$$

Let $\pi: T M \rightarrow M$ be the natural projection. Comparing with the curve $\gamma$ obtained by joining $x$ and $\pi(\theta)$ with a short curve, then following the curve $\pi \varphi_{t}(\theta)$ and then joining $\pi \varphi_{T}(\theta)$ and $y$ with a short curve, we have that for any given $\varepsilon>0$ and $T$ large enough

$$
S(T) \leq(m+\varepsilon) T+O(1)
$$

So

$$
\limsup _{T \rightarrow \infty} \frac{S(T)}{T} \leq(m+\varepsilon)
$$

Item (c) now follows from the fact due to Mañé [6] 3] that $m=-c(L)$.

Proof of Theorem [1. We begin with the last statement. Note that $L+k$ is bigger than zero if and only if $H(x, 0)<k$. Indeed

$$
H(x, p)=\max _{v \in T_{x} M}(p v-L(x, v))
$$

then

$$
H(x, 0)=\max _{v \in T_{x} M}(-L(x, v))=-\min _{v \in T_{x} M}(L(x, v)) .
$$

So if $k$ is bigger than $-\inf L$, then $H^{-1}(-\infty, k)$ contains the zero section of $T^{*} M$.

Now define a new Hamiltonian $G$ on $T^{*} M$ minus the zero section such that $G$ takes the value $\mu$ on $H^{-1}(k)$ and such that $G(x, \lambda p)=\lambda^{2} G(x, p)$ for all positive $\lambda$. Since $G$ is positively homogeneous of degree two and convex in $p$, it follows that the Legendre transform $F$ associated to $G$ is the square of a Finsler metric.

Since by definition $G^{-1}(\mu)=H^{-1}(k)$, it follows that the Hamiltonian flows of $G$ and $H$ coincide up to reparameterization on the energy level $G^{-1}(\mu)=H^{-1}(k)$ and therefore the Euler-Lagrange solutions of $L$ with energy $k$ are reparameterizations of geodesics of $\sqrt{F}$.

We claim that for an appropriate choice of $\mu$ and if $E(x, v)=k$, then

$$
\sqrt{F(x, v)}=L+k \text {. }
$$

It is proved in [6, 3] that for $k$ greater than the critical value and for any $x, y$ in $M$ there exists $\gamma$ such that $A_{L+k}(\gamma)=\Phi_{k}(x, y)$. Moreover $\gamma$ is a solution of the Euler-Lagrange equation and has energy $k$. Also, if $k>c(L)$, every curve can be reparameterized to have energy $k$ and the Finsler length does not depend on the reparameterization. By the definitions of both $D_{F}$ and $\Phi_{k}$, we may restrict ourselves to curves with energy $k$ and Theorem 1 follows in this case.

Proof of the claim. Since $G$ is homogeneous of degree 2 it follows from Euler's formula that $F$ and $G$ take the same value at Legendre related points.

Define $\lambda(x, p)$ such that $H\left(x, \frac{p}{\lambda}\right)=k$; then $G(x, p)=\mu \lambda^{2}(x, p)$. We have

$$
\frac{\partial H}{\partial p}\left(x, \frac{p}{\lambda}\right) \lambda^{-1}-\frac{\partial H}{\partial p}\left(x, \frac{p}{\lambda}\right) \cdot p \lambda^{-2} \frac{\partial \lambda}{\partial p}=0
$$


and

$$
\frac{\partial G}{\partial p}=2 \mu \lambda \frac{\partial \lambda}{\partial p}
$$

Multiplying (21) by $2 \mu \lambda^{3}$ we then get

$$
\frac{\partial H}{\partial p}\left(x, \frac{p}{\lambda}\right) \cdot p \frac{\partial G}{\partial p}=2 G(x, p) \frac{\partial H}{\partial p}\left(x, \frac{p}{\lambda}\right) .
$$

Suppose now that $E(x, v)=k$ and let $P(x, v)=\partial L / \partial v$; then by definition we have

$$
\begin{aligned}
\lambda(x, P(x, v)) & =1, \\
G(x, P(x, v)) & =\mu, \\
\frac{\partial H}{\partial p}(x, P(x, v)) & =v,
\end{aligned}
$$

and so

$$
\begin{aligned}
\frac{\partial H}{\partial p}(x, P(x, v)) \cdot P(x, v) & =v \frac{\partial L}{\partial v} \\
& =L+k \\
& >0
\end{aligned}
$$

Hence from (3) we have

$$
\frac{\partial G}{\partial p}(x, P(x, v))=\frac{2 v}{v \cdot P(x, v)} .
$$

Since $\partial G / \partial p$ is homogeneous of degree one and from (4) $v \cdot P(x, v)$ is positive, we obtain

$$
\frac{\partial G}{\partial p}\left(x, \frac{1}{2} v \cdot P(x, v) P(x, v)\right)=v .
$$

So $v$ is related to $\frac{1}{2} v \cdot P(x, v) P(x, v)$ with respect to the Legendre transform of $F$. Hence

$$
\begin{aligned}
F(x, v) & =G\left(x, \frac{1}{2} v \cdot P(x, v) P(x, v)\right) \\
& =\frac{(v \cdot P(x, v))^{2}}{4} G(x, P(x, v)) \\
& =\frac{(v \cdot P(x, v))^{2}}{4} \mu .
\end{aligned}
$$

So if $\mu=4$,

$$
\sqrt{F(x, v)}=v \cdot \frac{\partial L}{\partial v}
$$

Now let $k$ be bigger than $c(L)$. Then by a corollary in [4] there exists a $C^{\infty}$ real valued function $f$ on $M$, such that $H\left(x, d f_{x}\right)<k$. Define as in [4] $H_{d f}(x, p)=$ $H\left(x, p+d f_{x}\right)$. The Hamiltonian flows are conjugate by $\psi(x, p)=\left(x, p-d f_{x}\right)$. The 
Legendre transformation $L_{d f}$ of $H_{d f}$ is

$$
\begin{aligned}
L_{d f}(x, v) & =\max _{p \in T_{x}^{*} M}\left(p v-H_{d f}(x, p)\right) \\
& =\max _{p \in T_{x}^{*} M}\left(p v-H\left(x, p+d f_{x}\right)\right) \\
& =\max _{p \in T_{x}^{*} M}\left(\left(p-d f_{x}\right) v-H(x, p)\right) \\
& =L(x, v)-d f_{x} v .
\end{aligned}
$$

It turns out that

$$
\begin{aligned}
E\left(L_{d f}\right) & =E(L), \\
c\left(L_{d f}\right) & =c(L), \\
\Phi_{k}\left(L_{d f}\right)(x, y) & =\Phi_{k}(L)(x, y)-f(y)+f(x) .
\end{aligned}
$$

So as the zero section is contained in $H_{d f}^{-1}(-\infty, k), L_{d f}+k$ is positive and there is a Finsler metric such that

$$
\Phi_{k}\left(L_{d f}\right)(x, y)=D_{F}(x, y) .
$$

So

$$
\Phi_{k}(L)(x, y)=D_{F}(x, y)+f(y)-f(x) .
$$

\section{REFERENCES}

[1] R. Abraham \& J. Marsden. Foundations of Mechanics. Addison-Wesley, 1985. MR 81e:58025

[2] V.I. Arnold. Mathematical Methods of Classical Mechanics. Graduate Texts in Math., 60. Springer, 1989. MR 96c:70001

[3] G. Contreras, J. Delgado, R. Iturriaga, Lagrangian flows: the dynamics of globally minimizing orbits II, Bol. Soc. Bras. Mat. Vol. 28, N.2, (1997) 155-196. MR 98i:58093

[4] G. Contreras, R. Iturriaga, G. P. Paternain, M. Paternain. Lagrangian graphs, minimizing measures and Mañé's critical values. Geometric and Functional Analysis Vol. 8 (1998) 788809. MR 99f:58075

[5] M. Gutzwiller. Chaos in Classical and Quantum Mechanics New York. Springer Verlag (1990). MR 91m:58099

[6] R. Mañé, Lagrangian flows: the dynamics of globally minimizing orbits, International Congress on Dynamical Systems in Montevideo (a tribute to Ricardo Mañé), F. Ledrappier, J. Lewowicz, S. Newhouse eds., Pitman Research Notes in Math. 362 (1996) 120-131. Reprinted in Bol. Soc. Bras. Mat. Vol 28, N. 2, (1997) 141-153. MR 98i:58092

[7] J. Mather. Action minimizing invariant measures for positive definite Lagrangian systems. Math. Z. 207, (1991), no. 2, 169-207. MR 92m:58048

[8] R.T. Rockafellar. Convex Analysis. Princeton Landmarks in Math. Princeton. Princeton University Press (1997). MR 97m:49001

Cimat, A.P. 402, 36000, Guanajuato. Gto., México

E-mail address: renato@fractal.cimat.mx

Instituto de Matemáticas, UnAM, Ciudad Universitaria, C. P. 04510, México, DF, MÉXICO

E-mail address: hector@matem.unam.mx 